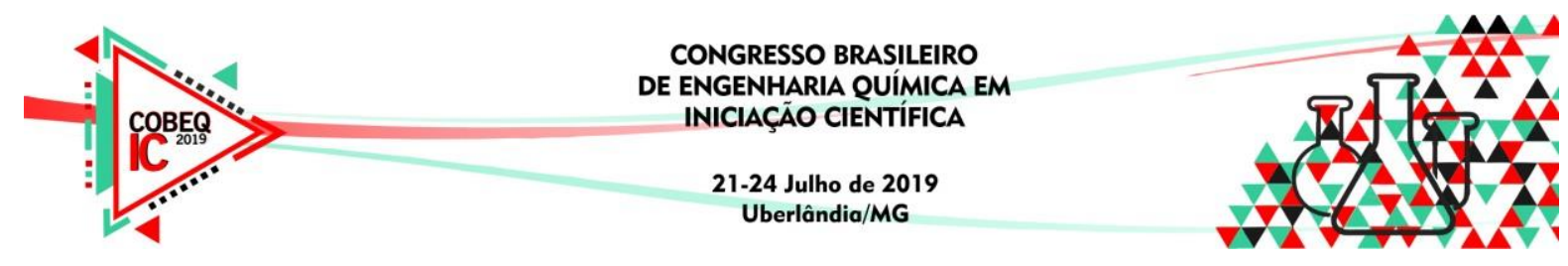

\title{
INFLUÊNCIA DA ARGILA ORGANOFÍLICA NAS PROPRIEDADES DE FLUIDOS DE PERFURAÇÃO MICROEMULSIONADOS
}

\author{
G. L. R. LEAL*, P. A. N. COSTA, A. C. B. JUNIOR, A. I. C. GARNICA, F. D. S. \\ CURBELO \\ Universidade Federal da Paraíba, Centro de Tecnologia, Departamento de Engenharia \\ Química \\ *E-mail para contato: giovannalais1998@ hotmail.com
}

\begin{abstract}
RESUMO - Um dos fatores mais importantes para que a perfuração de um poço de petróleo seja bem sucedida é a composição do fluido de perfuração. Portanto, é essencial que esses fluidos sejam formulados de modo a garantir a eficiência e segurança da perfuração do poço. Este trabalho teve como objetivo analisar a influência de duas argilas, uma delas organofilizada, na composição dos fluidos de perfuração microemulsionados, denominados FP1 e FP2. Os resultados obtidos mostraram que a argila bentonita organofílica desempenhou um papel importante na diminuição dos parâmetros reológicos e no volume de filtrado do FP2.
\end{abstract}

\section{INTRODUÇÃO}

A perfuração, uma das etapas da exploração de óleo e gás, é uma atividade que só pode ser realizada graças aos fluidos de perfuração que, segundo o Instituto Americano de Petróleo (API), são definidos como fluidos circulantes capazes de tornar a operação de perfuração viável.

Os aditivos exercem funções fundamentais na formulação do fluido. As argilas, um dos aditivos utilizados, são sólidos ativos que, de acordo com Thomas (2001), têm a função de viscosificar o fluido. Dessa forma, o presente trabalho teve como objetivo avaliar a influência da utilização de dois tipos de argila, a Closite 20A e a Bentonita Organofílica, nas propriedades dos fluidos microemulsionados.

\section{METODOLOGIA}

\subsection{Obtenção do diagrama ternário}

Para a construção do diagrama ternário, foram utilizados: um tensoativo não iônico NP100, óleo de pinho comercial como fase oleosa e uma solução aquosa de glicerina, na proporção 1:1, como fase aquosa.

O diagrama de fases ternário foi obtido utilizando a metodologia de titulação da fração mássica, que consiste em fixar a proporção de dois componentes do diagrama e titular com o terceiro componente até o surgimento da região monofásica, determinando, em seguida, a sua fração mássica. 


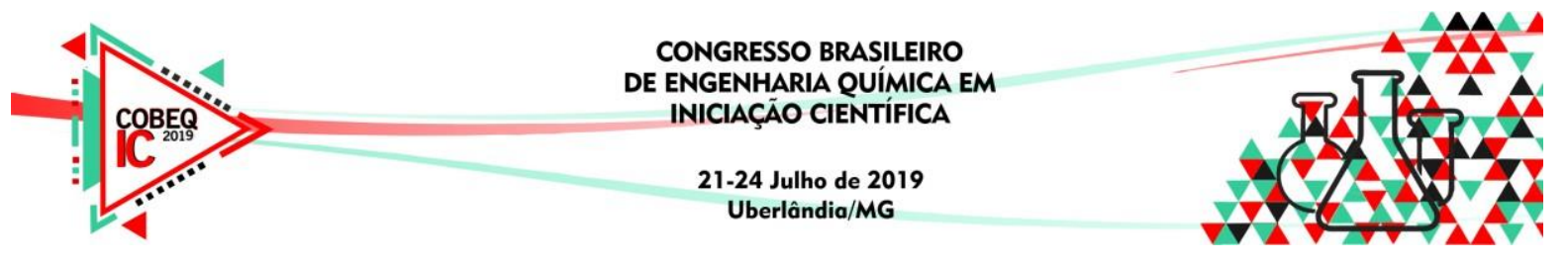

\subsection{Preparação dos fluidos de perfuração}

Foram preparados dois fluidos, FP1 e FP2, ambos base água, variando apenas a argila utilizada. As duas amostras de fluido foram preparadas a partir de $300 \mathrm{~g}$ de microemulsão, onde foram inseridos os aditivos, sob agitação constante, a uma velocidade de $620 \mathrm{rpm}$, em agitador Hamilton Beach, respeitando um intervalo de 10 minutos entre cada adição.

A adição dos componentes ocorreu na seguinte ordem: 10,01 g de cal, $4 \mathrm{~g}$ de argila (closite 20A para o FP1 e bentonita organofílica para o FP2), 1,01 g de HP-amido, 0,2 g de goma xantana, $80 \mathrm{~g}$ de barita e, por último, $450 \mathrm{~g}$ de água.

\subsection{Determinação dos parâmetros reológicos e massa específica}

O ensaio reológico foi realizado em viscosímetro Fann VG 35A, com o objetivo de determinar a viscosidade aparente (VA), a viscosidade plástica (VP), o limite de escoamento (LE) e as medidas de força gel inicial (G0) e força gel final (Gf) do fluido de perfuração. VA é o valor obtido na leitura a $600 \mathrm{rpm}$ dividido por 2, dada em $\mathrm{cP}$. VP é a diferença das leituras realizadas a $600 \mathrm{rpm}$ e a $300 \mathrm{rpm}$, dada também em cP. LE é a diferença entre a VP e a leitura em $300 \mathrm{rpm}$, dada por $\mathrm{N} / \mathrm{m}^{2}$.

Para a determinação das forças géis, é colocado uma rotação de $600 \mathrm{rpm}$ no viscosímetro durante 1 minuto, então muda-se para $3 \mathrm{rpm}$ e desliga-o. Espera-se o tempo de repouso indicado para cada uma das forças géis, 10 segundos para G0 e 10 minutos para Gf e, em seguida, o viscosímetro é ligado e é feita a leitura da máxima deflexão. As massas específicas dos fluidos de perfuração, FP1 e FP2, em $\mathrm{g} / \mathrm{cm}^{3}$, foram determinadas em uma balança de lama Fann, Modelo 140.

\subsection{Volume de Filtrado}

O volume de filtrado foi realizado através da filtração estática em um Filtro Prensa API Pressurizado, no qual foi aplicada, durante 30 minutos, uma pressão constante de 100 psi com ar comprimido à temperatura de, aproximadamente, $26^{\circ} \mathrm{C}$. Com o auxílio de uma proveta, foi possível determinar o volume de filtrado após 30 minutos do início da aplicação da pressão.

\subsection{Permeabilidade do reboco}

A permeabilidade do reboco foi determinada através da Equação 1. (Lomba, 2010):

$$
\mathrm{K}=\mathrm{Q}_{\mathrm{f}} \varepsilon \mu 8,9510^{-5}
$$

Em que: $\mathrm{K}$ é a permeabilidade, em $\mathrm{mD} ; \mathrm{Q}_{\mathrm{f}}$ é o volume de filtrado, em $\mathrm{cm}^{3}$; $\varepsilon$ é a espessura do reboco, em mm e $\mu$ é a viscosidade do fluido, em cP.

\section{RESULTADOS E DISCUSSÃO}

\subsection{Microemulsões}

O ponto de microemulsão escolhido, em fração mássica, foi selecionado com o objetivo de diminuir o custo do fluido de perfuração, reduzindo a quantidade de tensoativo e aumentando a proporção da fase aquosa. 


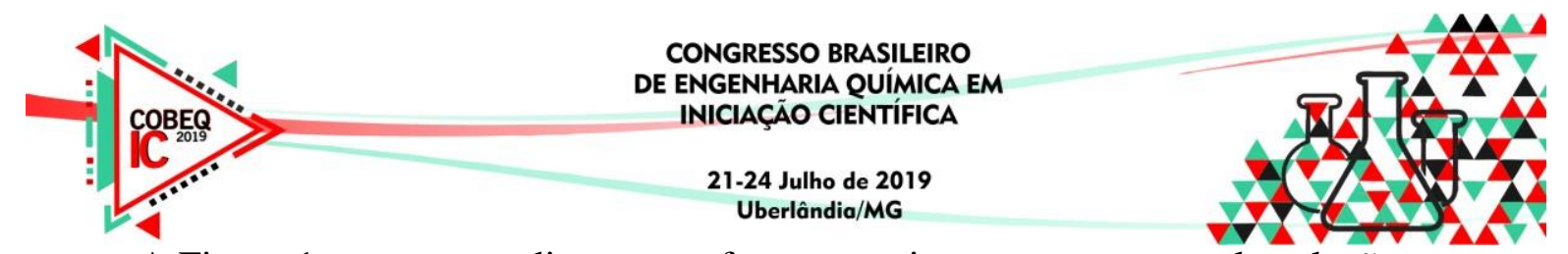

A Figura 1 apresenta o diagrama referente ao sistema composto pela solução aquosa, o tensoativo e o óleo.

Figura 1 - Diagrama referente ao sistema composto por solução aquosa de glicerina 1:1, NP100 e óleo de pinho.

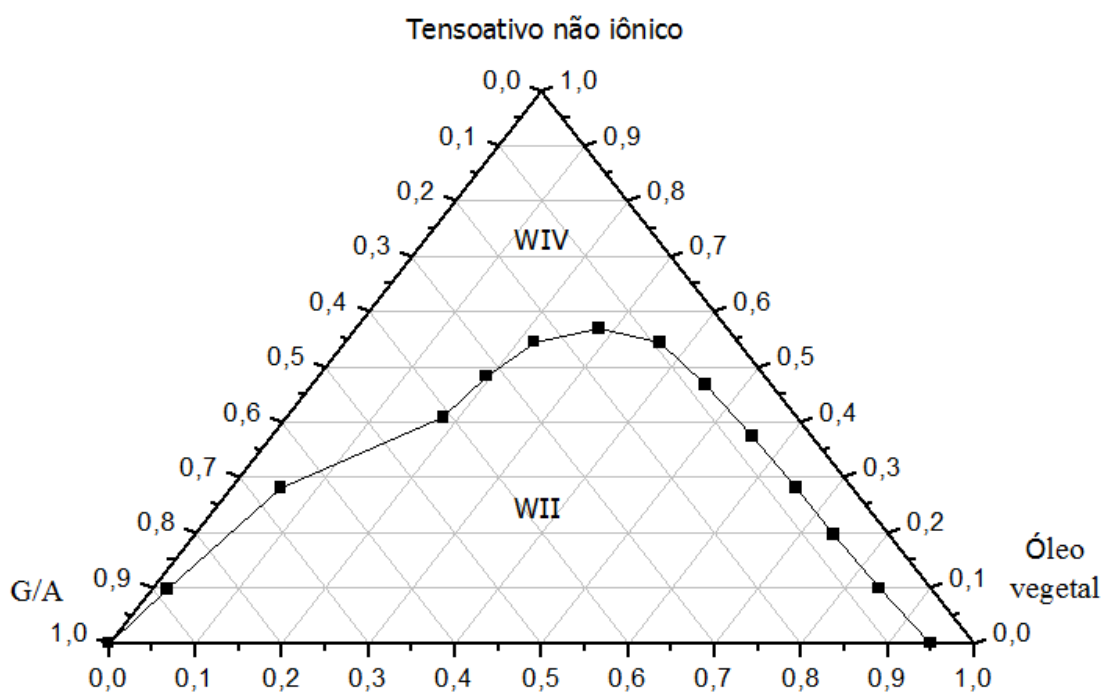

O ponto de microemulsão que foi estabelecido tem composição de $55 \%, 5 \%$ e $40 \%$ para fase aquosa, oleosa e de tensoativo, respectivamente.

\subsection{Estudo Reológico}

Inicialmente, foram determinadas, as massas específicas dos fluidos de perfuração formulados. O valor foi de, aproximadamente, $0,97 \mathrm{~g} / \mathrm{cm}^{3}$ para $\mathrm{FP} 1$ e $1,09 \mathrm{~g} / \mathrm{cm}^{3}$ para FP2. O pH foi determinado utilizando o pHmetro (TECNOPON mPA 210), resultando em 8,32 para FP1 e 8,20 para FP2. Posteriormente, foram realizados os ensaios reológicos nos fluidos de perfuração.

A Tabela 1 apresenta os parâmetros reológicos referentes aos fluidos de perfuração microemulsionados. Observando a Tabela 1, verifica-se que a presença da argila organofílica no FP2 provocou uma diminuição em todos os parâmetros reológicos estudados.

Tabela 1 - Parâmetros reológicos referentes aos fluidos de perfuração FP1 e FP2

\begin{tabular}{|c|c|c|c|c|c|}
\hline \multicolumn{5}{|c|}{ Parâmetros reológicos } \\
\hline Fluido & VP (cP) & VA (cP) & LE (lbf/100ft $\left.{ }^{\mathbf{~}}\right)$ & $\mathbf{G}_{\mathbf{0}}\left(\mathbf{l b f} / \mathbf{1 0 0 f t}^{\mathbf{2}}\right)$ & $\mathbf{G}_{\mathbf{f}}\left(\mathbf{l b f} / \mathbf{1 0 0 f \mathbf { f } ^ { \mathbf { 2 } } )}\right.$ \\
\hline FP1 & 108 & 133,5 & 51 & 7 & 12 \\
\hline FP2 & 105 & 118,8 & 27,5 & 4 & 5 \\
\hline
\end{tabular}

Os valores da massa específica, pH, VA e VP dos fluidos estão dentro das especificações exigidas pela norma PETROBRAS N-2604 (1998), que foi baseada na API Spec 13A (1993). 


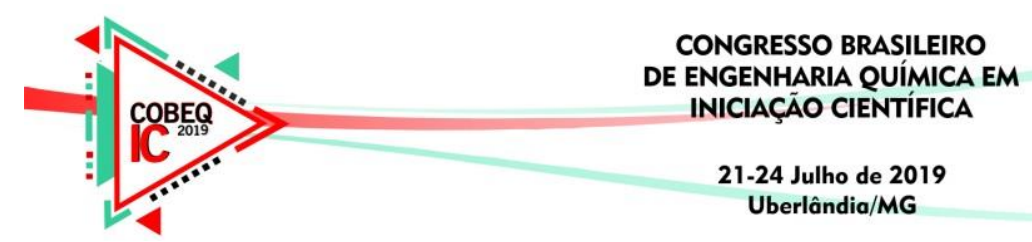

Figura 2 - Curva de fluxo.

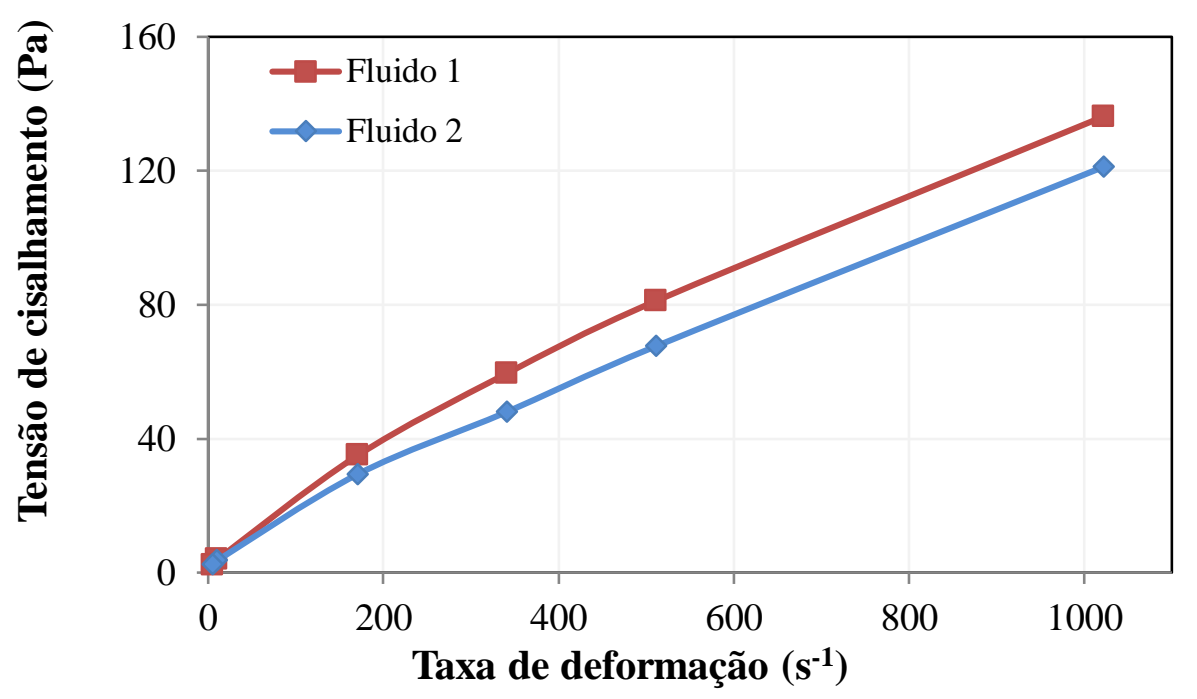

Figura 3 - Log $(\mu)$ em função de $\log (\gamma)$.

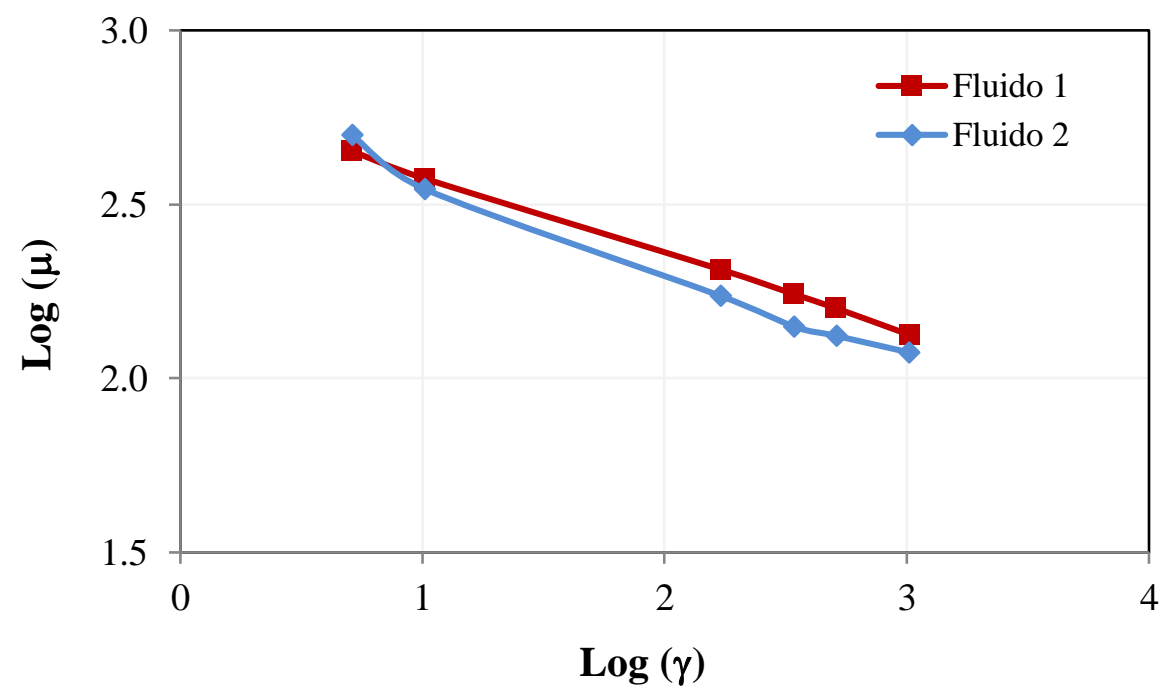

Ao analisar as Figura 2 e 3, nota-se que os dois fluidos de perfuração são não newtonianos e apresentam uma pequena tensão de cisalhamento inicial. Os modelos de comportamento de fluidos mais usuais apresentados na literatura, para este caso, são: modelo de Bingham ou plástico ideal e o modelo de Herschel-Bulkley, também conhecido como fluido de potência com limite de escoamento, ou fluido de potência modificado.

A principal diferença entre esses dois modelos é a relação entre a tensão de cisalhamento e a taxa de deformação que, para o modelo de Bingham é linear e para o de Herschel-Bulkley é não linear. Dessa forma, o modelo de Herschel-Bulkley foi o que melhor ajustou aos comportamentos apresentados. Os parâmetros deste modelo, calculados para FP1 e FP2, estão apresentados na Tabela 2. 


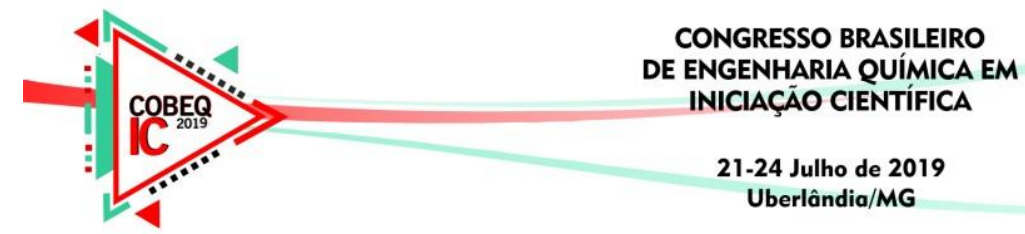

Tabela 2 - Parâmetros reológicos do modelo de Herschel-Bulkley

\begin{tabular}{|c|c|c|c|}
\hline Fluido & $\boldsymbol{\tau}_{\mathbf{0}}\left(\mathbf{N} / \mathbf{m}^{\mathbf{2}}\right)$ & $\mathbf{n}$ & $\mathbf{K}\left(\mathbf{P a} \cdot \mathbf{s}^{\mathbf{n}}\right)$ \\
\hline FP1 & 0,5 & 0,8221 & 0,2220 \\
\hline FP2 & 1,7 & 0,9304 & 0,2036 \\
\hline
\end{tabular}

Através da análise da Tabela 2, percebe-se que o FP1 apresentou maior índice de consistência $(\mathrm{K})$, indicando que oferece maior resistência ao escoamento, resultado que confirma o fato da viscosidade aparente ser maior para este fluido, conforme visto na Tabela 1.

De acordo com a Figura 3, a viscosidade diminui à medida que a taxa de cisalhamento aumenta. Este comportamento além de esperado é importante para o fluido de perfuração, pois com o aumento da velocidade de escoamento do fluido dentro da coluna de perfuração, a taxa de cisalhamento aumenta e, consequentemente, a viscosidade do fluido diminui, já que dentro da coluna o fluido necessita de uma certa facilidade para escoar.

\subsection{Volume de filtrado e permeabilidade do reboco}

O volume de filtrado no FP1 foi de 8,4 mL, e no FP2, 5,5 mL. Estes valores estão de acordo com os resultados obtidos por Martins et al. (2017). A espessura do reboco, medida de forma grosseira com o auxílio de um paquímetro, foi de 2,5 $\mathrm{mm}$ para o FP1 e 1,1 $\mathrm{mm}$ para o FP2. O resultado da permeabilidade do reboco obtida a partir da equação 1, foi de aproximadamente $0,25 \mathrm{mD}$ para o FP1 e $0,06 \mathrm{mD}$ para o FP2.

Figura 4 - Volume de filtrado em função do tempo.

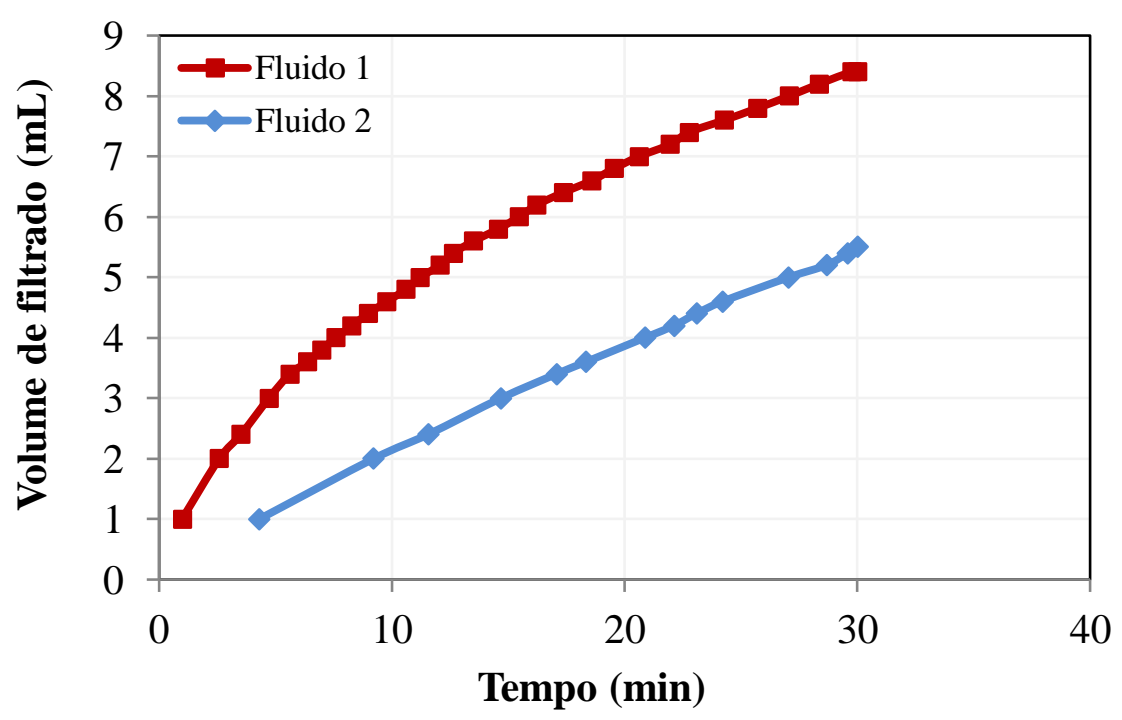




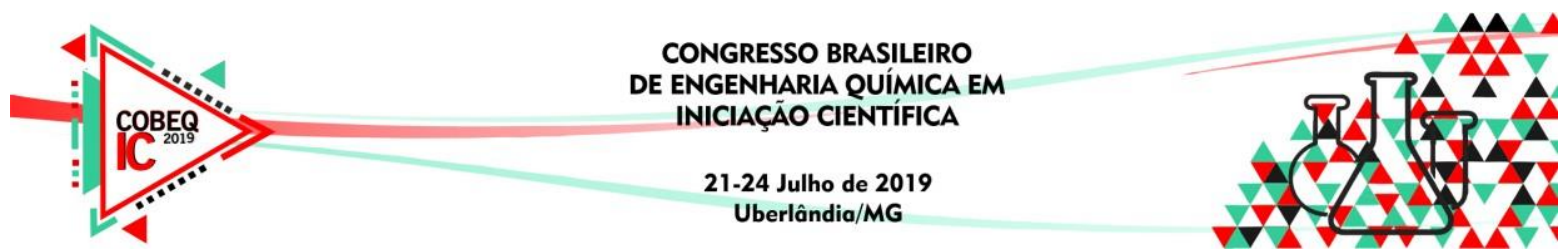

Por meio dos resultados obtidos, foi possível observar que a adição da argila organofílica ocasionou uma redução de quase $35 \%$ no volume de filtrado. Este resultado comprova a menor permeabilidade do reboco para o FP2, ou seja, ele mantém o poço mais estável, minimizando a invasão do filtrado e diminuindo os riscos de ocorrer prisão diferencial, já que a formação do seu reboco é menos espessa.

\section{CONCLUSÕES}

A argila organofílica presente no FP2 promoveu uma diminuição em todos os parâmetros reológicos estudados, acarretando uma menor resistência do fluido para reiniciar seu escoamento após determinado tempo de repouso.

A formulação do fluido FP2, com a organofilização da bentonita, diminuiu a invasão do filtrado, garantindo, assim, sua estabilidade e seu uso em aplicações petrolíferas com menos risco de ocorrência de prisão diferencial. Sendo assim, a bentonita organofílica apresentou resultados satisfatórios na formulação do fluido de perfuração.

\section{REFERÊNCIAS}

API. American Petroleum Institute, Specification for Drilling Fluid Materials. Washington, 1993.

LOMBA, R. Fundamentos de filtração e controle das propriedades de filtração. Report, 2010.

MARTINS, J. S.; NASCIMENTO, R. C. A. M.; AMORIM, L.V. Influência da adição de lubrificantes e sólidos inertes nas propriedades físicas de fluidos de perfuração argilosos. São Paulo, 2017.

THOMAS, J. E. Fundamentos de Engenharia de Petróleo. Editora Interciência, 2001. 

INICIAÇÃO CIENTÍFICA

21-24 Julho de 2019

Uberlândia/MG

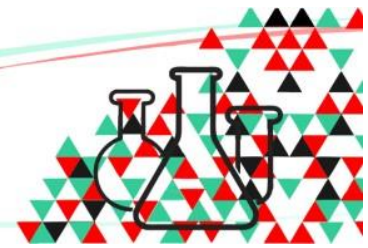

